\title{
Statistical Description of Eigenfunctions in Chaotic and Weakly Disordered Systems beyond Universality
}

\author{
Juan Diego Urbina* \\ Institute for Physics of Complex Systems, The Weizmann Institute of Science, 76100 Rehovot, Israel \\ Klaus Richter \\ Institut für Theoretische Physik, Universität Regensburg, 93040 Regensburg, Germany \\ (Received 21 May 2005; revised manuscript received 7 September 2006; published 22 November 2006)
}

We present a semiclassical approach to eigenfunction statistics in chaotic and weakly disordered quantum systems which goes beyond random matrix theory, supersymmetry techniques, and existing semiclassical methods. The approach is based on a generalization of Berry's random wave model, combined with a consistent semiclassical representation of spatial two-point correlations. We derive closed expressions for arbitrary wave-function averages in terms of universal coefficients and sums over classical paths, which contain, besides the supersymmetry results, novel oscillatory contributions. Their physical relevance is demonstrated in the context of Coulomb blockade physics.

DOI: 10.1103/PhysRevLett.97.214101

Since Chladni's famous experiments on vibrating plates precisely two centuries ago, the morphology of eigenfunctions in wave (mechanical) systems has aroused curiosity [1]. In particular, the statistical description of eigenfunctions in quantum (or wave) systems with diffusive or chaotic classical (or ray) dynamics has been an intensive research field for more than 20 years [2]. Besides the pure academic interest, the study of the spatial properties and the morphology of irregular eigenfunctions provides specific theoretical input for experimentally relevant quantities in a wide range of disciplines [1] including optics, acoustics, oceanography [3], quantum chaos, and mesoscopic condensed matter [4,5]. In the latter, for instance, the conductance of quantum dots is affected by spatial wave function correlations [6].

In the semiclassical limit [7], characterized by typical classical actions $S_{\mathrm{cl}}$ much larger than Planck's constant, i.e., $\hbar_{\mathrm{eff}} \equiv \hbar / S_{\mathrm{cl}} \ll 1$, statistical measures for the spatial structure of eigenfunctions can be deduced from purely classical quantities, though in a highly nontrivial way. The most spectacular example of such a connection is embodied in Berry's random wave model (RWM) [8]: eigenfunctions of classically chaotic systems possess the same statistical properties as Gaussian random fields with a universal spatial two-point correlation function [9].

More recently, deviations from universality have moved into the focus of research. A theory beyond the universal results of the RWM has been rigorously derived for disordered systems by an exact mapping of the quantum problem onto a supersymmetric field theory, the nonlinear sigma model [5]. Semiclassical in spirit, it expresses all results in terms of the classical diffusion propagator, and its success has motivated efforts to extend such methods to clean chaotic systems. Results of such (still conjectured) ballistic sigma models $(\mathrm{B} \sigma \mathrm{M})[5,10]$ are obtained by replacing the diffusion propagator for the disordered, metallic regime by a suitable ballistic counterpart.
PACS numbers: 05.45.Mt, 03.65.Sq

While deviations from universality in the supersymmetry approach have been usually taken as an indication of non-Gaussian statistics [11], a central result of this Letter is that such deviations in both clean and disordered systems in the metallic regime are actually perfectly consistent with local Gaussian statistics. Employing simple Gaussian integrations, we provide closed expressions for general wave function averages for chaotic systems in the semiclassical regime in terms of universal coefficients and sums over classical paths. We demonstrate that an arbitrary average contains, besides the universal (RWM) result, a systemdependent contribution (e.g., from confinement) composed of a smooth (diagonal) and an oscillatory part. We resolve the apparent contradiction to the supersymmetry techniques by showing how the results of the $\mathrm{B} \sigma \mathrm{M}$ beyond universality are retained from our results by neglecting all oscillatory contributions. This finding for chaotic dynamics is used to extend our method to the metallic regime of disordered systems, predicting, e.g., nonuniversal features for Coulomb blockade conductance peak statistics.

Defining statistical averages. - We consider (correlation) functions $F(\vec{\psi})=F\left(\psi\left(\vec{r}_{1}\right), \ldots, \psi\left(\vec{r}_{f}\right)\right)$ depending on the values of a wave function $\psi$ at $f$ different positions. Upon varying the index $n$ of eigenfunctions $\psi_{n}, F\left(\vec{\psi}_{n}\right)$ will exhibit fluctuations. Its spectral average at energy $e$ (indicated by calligraphic letters) is naturally defined as

$$
\mathcal{F}=\rho_{\epsilon}^{-1}(e) \sum_{n} w_{\epsilon}\left(e-e_{n}\right) F\left(\vec{\psi}_{n}\right)
$$

by means of the window function $w_{\epsilon}(x)$ with center at $x=$ 0 and width $\epsilon$. The normalization $\rho_{\epsilon}(e)=\sum_{n} w_{\epsilon}\left(e-e_{n}\right)$ is the density of states smoothed by $w_{\epsilon}$. Any approach to eigenfunction statistics aims at calculating $\mathcal{F}$ without complete knowledge of the exact eigenfunctions.

We achieve this through a Gaussian statistical assumption by representing $\mathcal{F}$ by means of a covariance matrix. 
Then a semiclassical approach is used by expressing the covariance matrix as a sum over classical interfering paths and by further expansions in powers of $\hbar_{\text {eff }}$.

Local Gaussian conjecture. - The position-dependent (local) Gaussian average of $F$ is equivalently given by

$$
\begin{aligned}
\mathcal{F}^{G} & =\int F(\vec{\psi}) \frac{e^{-(1 / 2) \vec{\psi} \cdot \mathbf{R}^{-1} \vec{\psi}}}{\sqrt{(2 \pi)^{f} \operatorname{det} \mathbf{R}}} d \vec{\psi}, \\
& =\int \bar{F}(\vec{\alpha}) \frac{e^{-(1 / 2) \vec{\alpha} \cdot \mathbf{R} \vec{\alpha}}}{\sqrt{(2 \pi)^{f}}} d \vec{\alpha} .
\end{aligned}
$$

Here $\bar{F}(\vec{\alpha})$ is the Fourier transform of $F(\vec{\psi})$ and $\mathbf{R}(e)=\mathbf{R}$ is the position-dependent covariance matrix defined through spatial two-point correlations as entries:

$$
R_{i, j}=\rho_{\epsilon}^{-1}(e) \sum_{n} w_{\epsilon}\left(e-e_{n}\right) \psi_{n}\left(\vec{r}_{i}\right) \psi_{n}\left(\vec{r}_{j}\right) .
$$

The local Gaussian conjecture states that eigenfunctions of a classically chaotic system behave locally like Gaussian random fields, i.e., $\mathcal{F}=\mathcal{F}^{G}$. This corresponds to transforming the discrete average over eigenstates in Eq. (1) into a continuous one over all possible states with a Gaussian distribution uniquely fixed by the systemdependent covariance matrix. This form of the conjecture is a natural generalization of ideas in Refs. [8,9,12]. It encodes all effects beyond universality into $\mathbf{R}$ while keeping all the advantages of Gaussian averaging.

Semiclassical covariance matrix. - According to quantum ergodicity, $R_{i, j} \rightarrow \delta_{i, j} / A(e)$ as $\hbar_{\text {eff }} \rightarrow 0$ [8] for twodimensional systems where $A(e)$ is the classically allowed area at energy $e$. In the semiclassical regime, quantum fluctuations appear as oscillations around this classical background. To make this separation explicit, we express numerator and denominator of $R_{i, j}$ through the semiclassical Green function. We obtain [12]

$$
R_{i, j}=\frac{1}{A(e)} \frac{\delta_{i, j}+\tilde{R}_{i, j}}{1+\tilde{\rho}_{\epsilon}(e)},
$$

where

$$
\begin{aligned}
\tilde{R}_{i, j}= & -\delta_{i, j}+J_{0}\left(k(e)\left|\vec{r}_{i}-\vec{r}_{j}\right|\right) \\
& +\sqrt{\frac{2 \hbar}{m^{2} \pi}} \sum_{\gamma_{i, j}} \bar{w}_{1 / \epsilon}\left(\frac{T_{\gamma_{i, j}}}{\hbar}\right)\left|D_{\gamma_{i, j}}\right|^{1 / 2} \cos \left(\frac{S_{\gamma_{i, j}}}{\hbar}\right)
\end{aligned}
$$

is a sum over classical paths joining $\vec{r}_{i}$ with $\vec{r}_{j}$ at fixed energy $e$. In Eq. (5), $\tilde{\rho}_{\epsilon}(e)=\int \tilde{R}_{i, i} d \vec{r}_{i}$ is the oscillatory part of the density of states (in units of the inverse mean level spacing). It is semiclassically expressed through the Gutzwiller trace formula [7]. The Bessel function $J_{0}$ with $k(e)=\sqrt{2 m\left[e-V\left(\left(\vec{r}_{i}+\vec{r}_{j}\right) / 2\right)\right]} / \hbar$ is well approximated (in billiards it is exact) by the contribution from the unique direct trajectory between $\vec{r}_{i}$ and $\vec{r}_{j}$. The sum in Eq. (6) is taken over all nondirect paths $\gamma_{i, j}$ with actions $S_{\gamma_{i, j}}\left(\vec{r}_{i}, \vec{r}_{j}, e\right)=\int_{\gamma_{i, j}} \vec{p} \cdot d \vec{r}$ and prefactors $D_{\gamma_{i, j}}$ which include the stability and topology of each path [7]. The
Fourier transform $\bar{w}_{1 / \epsilon}$ of the window function suppresses contributions from orbits with traversal time $T_{\gamma_{i, j}}=$ $\partial S_{\gamma_{i, j}} / \partial e$ larger than the characteristic time $\hbar / \epsilon$.

Semiclassical expansion. $-\tilde{R}_{i, j}$ and $\tilde{\rho}(e)$ are oscillatory functions of position and energy with amplitudes scaling as $\hbar_{\text {eff }}^{1 / 2}$ and $\hbar_{\text {eff }}$, respectively. Hence we can expand the covariance matrix elements in Eq. (5): $R_{i, j}=A(e)^{-1}\left[\delta_{i, j}+\right.$ $\left.\tilde{R}_{i, j}-\delta_{i, j} \tilde{\rho}_{\epsilon}(e)\right]$. This form allows, after inclusion into Eq. (3), for a consistent expansion of $\mathcal{F}$ in $\hbar_{\text {eff }}$ up to second order in $\tilde{R}$ and first order in $\tilde{\rho}$ : We find, neglecting terms of order $\hbar_{\mathrm{eff}}^{3 / 2}$,

$$
\begin{aligned}
\mathcal{F}= & \int \bar{F}(\vec{\alpha}) \frac{e^{-(\vec{\alpha} \cdot \vec{\alpha} / 2 A(e))}}{(2 \pi)^{f / 2}} \\
& \times\left[1-\frac{\vec{\alpha} \cdot\left(\tilde{\mathbf{R}}-\tilde{\rho}_{\epsilon}(e)\right) \vec{\alpha}}{2 A(e)}+\frac{(\vec{\alpha} \cdot \tilde{\mathbf{R}} \vec{\alpha})^{2}}{8 A(e)^{2}}\right] d \vec{\alpha} \\
= & \mathcal{F}^{(0)}+\mathcal{F}^{(R 1)}+\mathcal{F}^{(\rho)}+\mathcal{F}^{(R 2)} .
\end{aligned}
$$

As an example, for the intensity distribution $I_{t}\left(\vec{r}_{i}\right)=\delta(t-$ $\left.A(e) \psi\left(\vec{r}_{i}\right)^{2}\right)$ we easily obtain

$$
I_{t}\left(\vec{r}_{i}\right)=\frac{e^{-(t / 2)}}{\sqrt{2 \pi t}}\left[1-\frac{\tilde{R}_{i, i}-\tilde{\rho}_{\epsilon}}{2} H_{2}\left(\sqrt{\frac{t}{2}}\right)+\frac{\tilde{R}_{i, i}^{2}}{8} H_{4}\left(\sqrt{\frac{t}{2}}\right)\right]
$$

with $H_{n}(x)$ the $n$th Hermite polynomial.

We note that the terms $\mathcal{F}^{(R 1)}$ and $\mathcal{F}^{(\rho)}$ in Eq. (7) (including corrections up to order $\hbar_{\text {eff }}^{1 / 2}$ and $\hbar_{\text {eff }}$ ) are purely oscillatory in energy, in view of Eq. (6) and the Gutzwiller trace formula. They represent general expressions for boundary-affected nonuniversal contributions (one main result of this Letter) and will be further addressed below in the context of Coulomb blockade.

For energy windows larger than $\epsilon_{\mathrm{Th}}=\hbar / \tau_{\mathrm{Th}}$, with $\tau_{\mathrm{Th}}$ the time of flight through the system, i.e., the ballistic Thouless time, all contributions to $\tilde{R}_{i, j}$ beyond the directpath term are damped out, and $\tilde{\rho}_{\mathrm{Th}}(e)=0$. In this universal regime the RWM predictions are consistent with random matrix theory (RMT). They are obtained from $\mathcal{F}^{(0)}$ and from $\mathcal{F}^{(R 1)}$ by inserting the direct-path contribution to $\tilde{R}_{i, j}$ [first line in Eq. (6)] into Eq. (7). The Gaussian and sigmamodel results coincide at this universal level $[11,13]$. However, the term $\mathcal{F}^{(R 2)}$ in Eq. (7) contains an additional important smooth contribution as we will discuss below.

Diagonal approximation and sigma model. -For energy windows smaller than $\epsilon_{\mathrm{Th}}$, system-dependent deviations appear, i.e., $\mathcal{F}=\mathcal{F}^{\mathrm{RWM}}+\mathcal{F}^{\text {SYS }}$, as soon as longer paths affected by the confinement contribute. In our Gaussian approach deviations $\mathcal{F}^{G, S Y S}$ from universality are obtained by including the nondirect contributions to $\tilde{R}_{i, j}$ [second line of Eq. (6)] into Eq. (7). Hence $\mathcal{F}^{G, S Y S}$ consists of coherent single and double sums over nondirect classical paths of increasing length. Deviations $\mathcal{F}^{\sigma \text {,SYS }}$ from universality in the $\mathrm{B} \sigma \mathrm{M}$ are, on the contrary, expressed through a purely classical object, the ballistic propagator [11], and it 
is by no means clear whether the two approaches for $\mathcal{F}^{\mathrm{SYS}}$ are consistent.

As a first application of our approach, we address this fundamental issue. The ballistic propagator $\Pi_{i, j}^{\text {ball }}(w)$ is constructed by projecting the resolvent of the classical Liouville equation, $\{H(\vec{r}, \vec{p}), \rho\}=i w \rho$ (where $\{\cdots\}$ is the Poisson brackets), onto configuration space at energy $e=p^{2} / 2 m$ (focusing for simplicity on billiard systems). To compare the local Gaussian and sigma-model approaches we express $\Pi_{i, j}^{\text {ball }}(w)$ through a sum over paths of finite length [14], $\Pi_{i, j}^{\mathrm{ball}}(w)=\Pi_{i, j}^{\mathrm{dir}}(w)+\sum_{\gamma_{i, j}} D_{\gamma_{i, j}} e^{i w T_{\gamma_{i, j}}}$, where the term $\Pi_{i, j}^{\mathrm{dir}}$ from direct paths (set to zero when $\left.\vec{r}_{i}=\vec{r}_{j}\right) \quad$ contains $D_{i, j}^{\mathrm{dir}}=m^{2} / p\left|\vec{r}_{i}-\vec{r}_{j}\right| \quad$ and $T_{i, j}^{\mathrm{dir}}=$ $m\left|\vec{r}_{i}-\vec{r}_{j}\right| / p$. The related energy-averaged version reads

$$
\tilde{\Pi}_{i, j}^{\text {ball }}(w)=\Pi_{i, j}^{\mathrm{dir}}(w)+\sum_{\gamma_{i, j}} D_{\gamma_{i, j}} \bar{w}_{1 / \epsilon}^{2}\left(T_{\gamma_{i, j}} / \hbar\right) e^{i w T_{\gamma_{i, j}}} .
$$

Clearly $\tilde{\Pi}_{i, j}^{\text {ball }}(w) \rightarrow \Pi_{i, j}^{\text {ball }}(w)$ for $\epsilon \ll \epsilon_{\mathrm{Th}}$.

Consider the contribution $\mathcal{F}^{(R 2)}$, Eq. (7), to $\mathcal{F}$, Eq. (2), explicitly given by $\mathcal{F}^{(R 2)}=\sum_{i, j, k, l} \mathcal{F}_{i, j}^{k, l} \tilde{R}_{i, j} \tilde{R}_{k, l}$. The universal coefficients (using $\partial_{i, j}^{k, l}=\partial^{4} / \partial \psi_{i} \partial \psi_{j} \partial \psi_{k} \partial \psi_{l}$ )

$$
\mathcal{F}_{i, j}^{k, l}=\left[\frac{A(e)}{2 \pi}\right]^{f / 2} \int F(\vec{\psi}) \partial_{i, j}^{k, l} e^{-[A(e) / 2] \vec{\psi} \cdot \vec{\psi}} d \vec{\psi}
$$

depend only on $A(e)$. In view of Eq. (6), the nonuniversal part of $\tilde{R}_{i, j} \tilde{R}_{k, l}$ is a double sum over terms with phases $\left[S_{\gamma_{i, j}}(e) \pm S_{\gamma_{k, l}}(e)\right] / \hbar$. For $(i, j)=(k, l)$ they cancel and yield the nonoscillatory, so-called diagonal part

$$
\left[\tilde{R}_{i, j} \tilde{R}_{i, j}\right]_{\text {diag }}^{\mathrm{SYS}}=\frac{2 \hbar}{m^{2} \pi} \sum_{\gamma_{i, j}} D_{\gamma_{i, j}} \bar{w}_{1 / \epsilon}^{2}\left(T_{\gamma_{i, j}} / \hbar\right) .
$$

Comparing Eqs. (9) and (11) shows that the smooth contribution to any average beyond the universal RWM is

$$
\mathcal{F}_{\text {diag }}^{\text {SYS }}=\frac{2 \hbar}{m^{2} \pi} \sum_{i, j} \mathcal{F}_{i, j}^{i, j}\left[\tilde{\Pi}_{i, j}^{\text {ball }}(0)-\Pi_{i, j}^{\mathrm{dir}}(0)\right] .
$$

To our knowledge, no general expression such as Eq. (12) has been derived within the $\mathrm{B} \sigma \mathrm{M}$; however, explicit calculations of $\mathcal{F}_{i, j}^{i, j}$ show agreement with the various specific $\mathrm{B} \sigma \mathrm{M}$ results available (moments of a wave function, distribution of intensities, two-energies four-point correlations [5], and two-point intensity distributions [15]). This implies that to leading order in the deviation from universality, the $\mathrm{B} \sigma \mathrm{M}$ corresponds to a Gaussian theory in diagonal approximation.

To give two examples, Eqs. (10) and (12) yield for the smooth part of the intensity distribution (8), $[\exp (-t / 2) / \sqrt{2 \pi t}]\left\{1+\left[\hbar /\left(2 \pi m^{2}\right)\right] H_{4}(\sqrt{t / 2}) \tilde{\Pi}_{i, i}^{\text {ball }}(0)\right\}$ and $(2 q-1) ! !\left\{1+\left[\hbar q(q-1) /\left(4 \pi m^{2}\right)\right] \tilde{\Pi}_{i, i}^{\text {ball }}(0)\right\}$ for its moments $I_{q}\left(\vec{r}_{i}\right) \equiv A(e)^{q} \psi^{2 q}\left(\vec{r}_{i}\right)$, in agreement with supersymmetry [16].

Here several remarks are due: (i) Most importantly, our method provides also the general leading-order deviation from universality for the body of averages in disordered systems in the metallic regime by simply replacing $\tilde{\Pi}_{i, j}^{\text {ball }}(w)$ by the diffusive propagator; (ii) there is not a single chaotic system where the exact ballistic propagator is known; hence the use of few classical paths to construct the smoothed version (9) renders the Gaussian theory better accessible for practical calculations; (iii) the direct-path contribution, known to be counted twice as an artifact of sigma-model calculations [11], is correctly incorporated in the present approach [the diagonal part beyond the RWM is given by $\tilde{\Pi}_{i, j}^{\text {ball }}(w)-\tilde{\Pi}_{i, j}^{\mathrm{dir}}(w)$ instead of $\tilde{\Pi}_{i, j}^{\text {ball }}(w)$ as in the $\left.\mathrm{B} \sigma \mathrm{M}\right]$; (iv) our results can be generalized for broken time-reversal symmetry by taking $\vec{\psi}$ in Eq. (2) as a complex vector and substituting $\psi_{n}\left(\vec{r}_{j}\right)$ by its complex conjugate in Eq. (4); our results further include the case of smooth potentials; (v) statistics involving different eigenfunctions at energies $e_{n}, e_{m}$ are treated by considering them as independent Gaussian fields and by expressing the results in terms of $\tilde{\Pi}_{i, j}^{\text {ball }}\left[w=\left(e_{n}-e_{m}\right) / \hbar\right]$.

Statistics and oscillations of Coulomb blockade conductance peaks. - As a further application of our approach we consider transport through a quantum dot weakly coupled to two leads. In this Coulomb blockade (CB) regime, characterized by the mean resonance width $\bar{\Gamma} \ll k_{B} T \ll$ $\Delta$, transport is mediated by resonant tunneling with related distinct conductance peaks. These CB peaks and fluctuations in their heights have been prominent objects of experiments [17]. The universal contribution to the conductance peak height distribution, derived in RMT [18] for one-channel leads, was extended to the multichannel case for ballistic quantum dots using the RWM [19] and for disordered systems using the sigma model [20].

We use our method to obtain nonuniversal effects in disordered quantum dots by first calculating the diagonal contribution to the conductance distribution in ballistic systems, and then substituting

$$
\frac{\hbar}{4 \pi m^{2}} \Pi_{i, j}^{\text {ball }} \rightarrow \frac{\delta_{i, j}}{\pi g_{0}} \ln \frac{L}{l}
$$

for a system of size $L$, white-noise disorder with mean free path $l$, and dimensionless conductance $g_{0}$.

In proceeding along these lines we consider two leads supporting one channel each, connected to the quantum dot at positions $\vec{r}_{1}$ and $\vec{r}_{2}$ with coupling strength $\alpha$; generalizations to more channels are straight forward. The height of the $n$th conductance peak is given by $G_{n}=\left(2 e^{2} / h\right) \times$ $(\alpha \Delta) /\left(\pi k_{B} T\right) g_{n}$ (see Refs. [18,19]), where

$$
g_{n}=A \frac{\psi_{n}^{2}\left(\vec{r}_{1}\right) \psi_{n}^{2}\left(\vec{r}_{2}\right)}{\psi_{n}^{2}\left(\vec{r}_{1}\right)+\psi_{n}^{2}\left(\vec{r}_{2}\right)}
$$

fluctuates with $n$. Assuming that no direct-path connects the leads, the conductance distribution has the form

$$
P(g)=P^{\mathrm{RMT}}(g)+P_{\text {diag }}^{\mathrm{SYS}}(g)+\text { oscillatory terms. }
$$

Here, the universal contribution is 


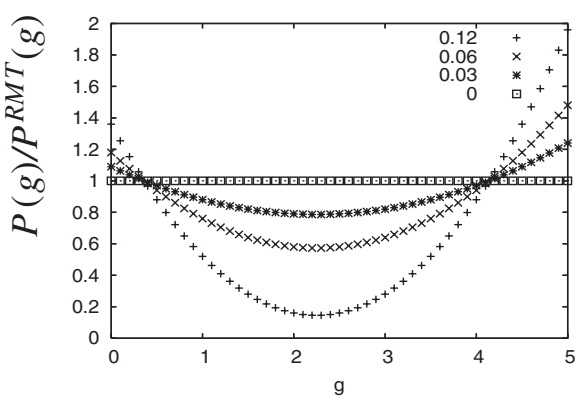

FIG. 1. Normalized Coulomb blockade conductance peak height distribution $P(g) / P^{\mathrm{RMT}}(g)$ for a disordered quantum dot [Eq. (19)]. Deviations from universality (squares) are depicted for values 0.12 (plus symbol), 0.06 (times symbol), and 0.03 (asterisk symbol) of the small parameter $\left(1 / \pi g_{0}\right) \ln (L / l)$.

$$
\begin{aligned}
P^{\mathrm{RMT}}(g) & =\frac{1}{\pi} \int \delta\left(g-2 \frac{\psi_{1}^{2} \psi_{2}^{2}}{\psi_{1}^{2}+\psi_{2}^{2}}\right) e^{-\left(\psi_{1}^{2}+\psi_{2}^{2}\right)} d \psi_{1} d \psi_{2} \\
& =\frac{\exp (-g / 2)}{\sqrt{2 \pi g}},
\end{aligned}
$$

and the nonuniversal part reads, following Eq. (12),

$$
P_{\text {diag }}^{\mathrm{SYS}}(g)=\frac{2 \hbar}{m^{2} \pi} \sum_{i=1}^{2} \sum_{j=1}^{2} P_{i, j}(g) \Pi_{i, j}^{\text {ball }}(0),
$$

with universal factors $P_{i, j}(g)$ obtained from Eq. (10) as

$$
\begin{aligned}
P_{i, j}(g)= & \frac{A^{2}}{4 \pi} \int \delta\left(g-2 \frac{\psi_{1}^{2} \psi_{2}^{2}}{\psi_{1}^{2}+\psi_{2}^{2}}\right) e^{-\left(\psi_{1}^{2}+\psi_{2}^{2}\right)} \\
& \times\left[H_{2}\left(\psi_{1}\right) H_{2}\left(\psi_{2}\right)\left(1-\delta_{i, j}\right)\right. \\
& \left.+H_{4}\left(\psi_{1}\right) \delta_{i, j}\right] d \psi_{1} d \psi_{2} .
\end{aligned}
$$

Equation (18) can be evaluated in closed form, and we find

$$
P(g)=P^{\mathrm{RMT}}(g)\left[1+\left(2 g^{2}-9 g+3\right) \frac{\ln (L / l)}{\pi g_{0}}\right]
$$

using Eq. (13). As summarized in Fig. 1, we find strong deviations from universality, even for very small values of the small parameter $\left(1 / \pi g_{0}\right) \ln (L / l)$.

Finally, we comment on the role of the oscillatory contributions to Eq. (7) in the context of CB. The leading-order oscillatory contribution, $\mathcal{F}^{(R 1)}$, to the conductance in clean systems, proportional to $\tilde{R}_{i, j}$, gives rise to well-defined peaks in the power spectra of the average conductance as a function of the wave number. As shown in Ref. [21], the peaks are located at the lengths $L_{\gamma}$ of the shortest classical paths $\gamma$ starting and ending at the leads, in perfect agreement with our results. The second-order oscillatory contribution, $\mathcal{F}^{(R 2)}$, predicted here for the first time, implies also the existence of peaks located at $L_{\gamma}-L_{\gamma^{\prime}}$, providing an explanation of the numerical observations of Ref. [21].

To summarize, we developed a local Gaussian approach for eigenfunction statistics to derive general expressions for both smooth and oscillatory effects beyond the universal RWM. Smooth contributions are shown to include existing results of the sigma model for both the ballistic and diffusive regime, illuminating the connection between the two methods beyond universality. We derived the conductance peak height distribution in disordered quantum dots, and found novel oscillatory contributions for the ballistic case, neglected both by the sigma model and previous semiclassical approaches, which mark further major deviations from universality.

We gratefully acknowledge conversations with C. Lewenkopf and A. Mirlin. This work was supported by the Deutsche Forschungsgemeinschaft (GRK 638). J. D. U. received additional support from the Minerva Center for Nonlinear Physics of Complex Systems (GIF 808/2003).

*Present address: Department of Physics, Universidad Nacional de Colombia, Ciudad Universitaria, Bogota, Colombia.

Electronic address: jdurbinag@unal.edu.co.

[1] See H.-J. Stöckmann, Quantum Chaos: An Introduction (Cambridge University Press, Cambridge, England, 1999), Chap. II.

[2] S. W. McDonald and A. N. Kaufman, Phys. Rev. Lett. 42, 1189 (1979); see, e.g., the reviews in Supersymmetry and Trace Formulae, edited by I. V. Lerner, J. P. Keating, and D. E. Khmelnitskii (Kluwer, New York, 1999).

[3] M.S. Longuet-Higgins, Phil. Trans. R. Soc. A 250, 157 (1957).

[4] C. W. J. Beenakker, Rev. Mod. Phys. 69, 731 (1997).

[5] A. D. Mirlin, Phys. Rep. 326, 259 (2000).

[6] Y. Alhassid, Rev. Mod. Phys. 72, 895 (2000).

[7] M.C. Gutzwiller, Chaos in Classical and Quantum Mechanics (Springer, New York, 1990).

[8] M. V. Berry, J. Phys. A 10, 2083 (1977).

[9] For RWM generalizations to the nonisotropic case, see M. V. Berry and H. Ishio, J. Phys. A 35, L447 (2002); W. E. Bies, N. Lepore, and E. J. Heller, J. Phys. A 36, 1605 (2003); J. D. Urbina and K. Richter, ibid. 36, L495 (2003); A. Bäcker and R. Schubert, J. Phys. A 35, 527 (2002).

[10] For a recent proposal, see K. B. Efetov, G. Schwiete, and K. Takahashi, Phys. Rev. Lett. 92, 026807 (2004).

[11] I. V. Gornyi and A. D. Mirlin, Phys. Rev. E 65, 025202 (2002).

[12] S. Hortikar and M. Srednicki, Phys. Rev. Lett. 80, 1646 (1998); L. Kaplan, ibid. 80, 2582 (1998).

[13] M. Srednicki, Phys. Rev. E 54, 954 (1996).

[14] M. Sieber, J. Phys. A 32, 7679 (1999).

[15] V. N. Prigodin and B. L. Altshuler, Phys. Rev. Lett. 80, 1944 (1998).

[16] Y. V. Fyodorov and A. D. Mirlin, Phys. Rev. B 51, 13403 (1995).

[17] A. M. Chang et al., Phys. Rev. Lett. 76, 1695 (1996); J. A. Folk et al., ibid. 76, 1699 (1996).

[18] R. A. Jalabert, A. D. Stone, and Y. Alhassid, Phys. Rev. Lett. 68, 3468 (1992).

[19] Y. Alhassid and C.H. Lewenkopf, Phys. Rev. Lett. 75, 3922 (1995).

[20] V. V. Prigodin, K. B. Efetov, and S. Iida, Phys. Rev. Lett. 71, 1230 (1993).

[21] E. E. Narimanov et al., Phys. Rev. B 64, 235329 (2001). 